\title{
Genome build information is an essential part of genomic track files
}

\author{
Chakravarthi Kanduri ${ }^{1,2}$, Diana Domanska ${ }^{1}$, Eivind Hovig ${ }^{1,3,4}$ and Geir Kjetil Sandve ${ }^{1,2^{*}}$ (D)
}

\begin{abstract}
Genomic locations are represented as coordinates on a specific genome build version, but the build information is frequently missing when coordinates are provided. We show that this information is essential to correctly interpret and analyse the genomic intervals contained in genomic track files. Although not a substitute for best practices, we also provide a tool to predict the genome build version of genomic track files.
\end{abstract}

\section{Background}

The data deluge that arose with the advent of highthroughput sequencing methods needs no introduction [1]. To enable reproducibility and reuse of the data, a community-driven common practice encourages researchers to deposit the generated data to public repositories [2, 3]. Larger research consortia that have generated data spanning billions of base pairs across a plethora of individuals, cell types and experimental conditions have also made their data public [4-6]. This good practice facilitates the reuse of the data to dig up further biological insights (e.g. see [7, 8]). However, the reuse of data in such a manner depends largely on the availability of metadata, which among other information describes the essential experimental and data processing details associated with the dataset $[9,10]$. Still, we find that sufficient metadata are often lacking for datasets in both public repositories and journal articles.

One fundamental, but surprisingly common, missing information element of genomic datasets is the genome build version that a dataset relates to. This is especially problematic for file formats solely containing genomic intervals. Just as the start and end positions (coordinates) in a BED file or GFF file do not provide any information without the knowledge of which chromosome the positions (offset) refers to, the chromosome and position together (genomic coordinates) also do not denote any meaningful location without the version of a

\footnotetext{
* Correspondence: geirksa@ifi.uio.no

'Department of Informatics, University of Oslo, 0316 Oslo, Norway

${ }^{2}$ K.G. Jebsen Coeliac Disease Research Centre, University of Oslo, 0318 Oslo,

Norway

Full list of author information is available at the end of the article
}

genome build. In other words, without the genome build information, the sequence coordinates would just be house numbers without a street name. Therefore, for files that exclusively provide data in the form of coordinates on a reference genome, such as BED, WIG or GFF, the genome build version is not only critical, but even a part of the data itself (they are actually data, not metadata).

Although the failure to supply the genome build information often occurs, the way this vital information is also being collected and stored is an equally big concern. Several of the public repositories and journals recommend the submission of a range of metadata (including genome build information) [9, 10], which are usually stored and provided in a separate file or web page (but not as an integral part of the data file itself). In other words, the common file formats that solely contain genomic intervals do not necessarily carry genome build information in any form (Table 1). Owing to the largely collaborative nature of genomics research (as of now), the data may travel between several computers back and forth during a project, thus requiring the documentation and explicit specification of genome build information every single time (e.g. in email or otherwise). However, this process is error prone and is prone to failure. Eventually, lack of genome build information could become a major time thief that may also potentially lead to erroneous data integrations. Here, we demonstrate the frequent isolation of genome build information from genomic track files and propose that this information should rather be an essential part of the data file itself. 
Table 1 Examples of detachment of genome build information for files downloaded from public repositories

\begin{tabular}{llll}
\hline File-hosting repository & $\begin{array}{l}\text { Genome build } \\
\text { information in } \\
\text { filename }\end{array}$ & $\begin{array}{l}\text { Genome build } \\
\text { information in } \\
\text { header }\end{array}$ & $\begin{array}{l}\text { Example } \\
\text { file }\end{array}$ \\
\hline UCSC & No & No & {$[24]$} \\
ENCODE & No & No & {$[25]$} \\
Roadmap Epigenomics & No & No & {$[26]$} \\
GEO & No & No & {$[27]$} \\
\hline
\end{tabular}

UCSC University of California Santa Cruz

\section{Methods and results}

\section{Extent of incompatibility between two genome build} versions

One of the common consequences of missing genome build information is the integration of genomic coordinates from two different genome build versions, which could largely be erroneous. To exemplify this error, we checked the extent of compatibility between two versions of human genome builds, hg19 and hg38. For this, we downloaded the size of both autosomes and sex chromosomes for hg19 from the University of California Santa Cruz (UCSC) database using fetchChromSizes [11] and segmented the chromosomes into $1-\mathrm{kb}$ bins, resulting in a total of $3,095,689$ bins. The genomic coordinates of the bins were then lifted over to hg38 using the UCSC liftover tool, where the conversion failed for 238,542 bins $(7.7 \%$ of total bins). For the bins that were common between both hg19 and hg38, we computed the distance between the midpoint of each bin on hg19 and its corresponding bin (after lift-over coordinates) on hg38 to know whether the genomic coordinates remained identical between genome builds. This revealed that only approximately 18,696 bins $(0.65 \%$ of the total number of bins) had identical genomic coordinates on both hg19 and hg38 for autosomes and sex chromosomes, and approximately $89.0 \%$ of the total bins were further apart than $30 \mathrm{~kb}$. Overall, integration of genomic intervals between these two builds would be erroneous for approximately $99.4 \%$ of total bins $(3,076,993,000 / 3,095,689,000$ bases $)$. Despite the large discrepancy, this problem could easily be overlooked when performing genome arithmetic operations because of the common sequence names and coordinates between different versions of genome builds; for example, see Fig. 1.

\section{Genome build information in a sample of repositories and journals}

We first determined whether genome build information is consistently supplied along with submissions to public repositories. As a representative example, we examined the records in the GEO and ENCODE

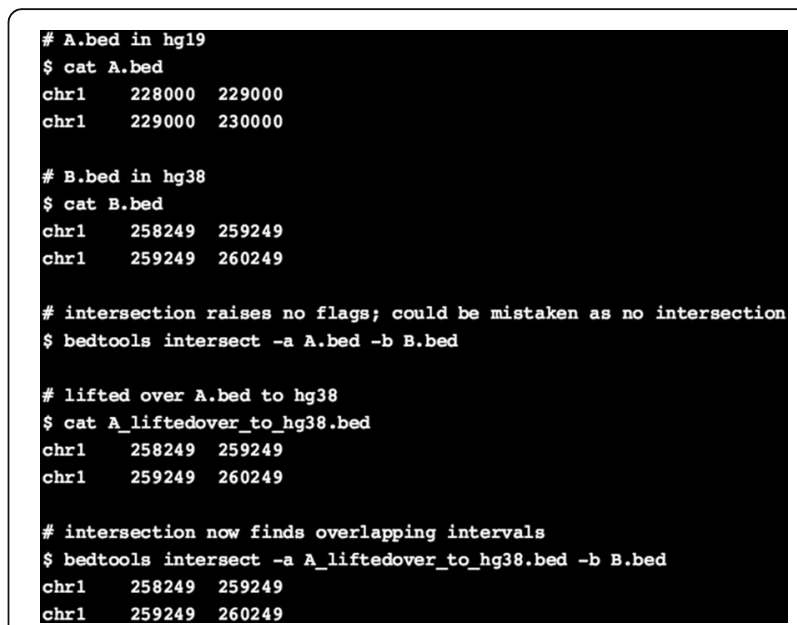

Fig. 1 Example of erroneous genome arithmetic operations because of integrating data from incompatible genome builds. The sequence intervals in A.bed (hg19) correspond to the intervals in B.bed (hg38). However, when genome arithmetic operations (like intersection) are performed in the absence of genome build information, there will be no overlap between the intervals in both the files, as the coordinates appear non-overlapping. If it is known that the files correspond to two different genome builds, lift-over of coordinates in one file would result in the detection of overlapping genomic intervals between both the files

databases with the following search criteria. In the GEO database, we examined all the records (one sample per series) that involved high-throughput sequencing submitted after 31 December 2008 for three species: Homo sapiens; Mus musculus; and Drosophila melanogaster. We then checked whether the data-processing section of metadata explicitly mentioned the genome build information, by case-insensitively searching for the following words:

\{hg17|hg18|hg19|hg38|grch36|grch37|grch38|build37.2|build37.1|build36.3 |ncbi35 $\mid$ ncbi36 $\mid$ ncbi37 $|\mathrm{mm} 8| \mathrm{mm} 9 \mid$ mm10|grcm38|bdgp6|bdgp5|bdgp5.25|build5.41|build5.3| build5|build4.1|dm6 $|\mathrm{dm} 3|$ ncbi $\}$. In the ENCODE database, we examined the metadata file of all records.

Around $23.0 \%$ of the queried series records did not contain the genome build information explicitly in the data-processing section of metadata in the GEO database (queried on 17 March 2017 according to the search criteria stated above), whereas all the relevant records in the ENCODE database contained genome build information in the metadata section.

Next, using a similar set of search criteria, we retrieved a total of 6155 articles across four journals. We then employed a series of filtering steps to shortlist articles that had a GEO accession ID corresponding to a highthroughput sequencing experiment, resulting in 332 articles. Of those, about $14.0 \%$ of the articles did not mention genome build information in the full text of the article, but it was mentioned in the metadata section of 
the GEO database. On the other hand, about $16.0 \%$ of the articles mentioned the genome build information in the full text of the article, but not in the GEO database, from where the data are usually downloaded by other researchers. Approximately $4.0 \%$ of the articles did not supply genome build information to either journal or repository (detailed in Additional file 1: Tables S1 and S2).

\section{Detachment of genome build information from genomic track files}

To demonstrate the extent of detachment of genome build information from data files, we next downloaded several files from public repositories and checked whether genome build information was carried along with the files in some form. First, we checked for the file formats BED and GFF that are attached as supplementary files to sample records in the GEO database. Again, after restricting the search criteria to three species (Homo sapiens, Mus musculus and Drosophila melanogaster), we retrieved 967 BED files and 2100 GFF files. We then checked whether the filenames or the header lines (we checked the first 50 lines) explicitly mentioned the genome build information. For this, we again searched for the names of specific genome builds of the three species listed above. Overall, while approximately $46.0 \%$ of the total BED files from the GEO database carried genome build information, only about $0.6 \%$ of the total GFF files carried it either in the filename or in the header (Table 2).

Further, we downloaded 26,503 BED narrowPeak files (from a total of 4775 records: Homo sapiens 3533; Mus musculus 994; and Drosophila melanogaster 248) from the ENCODE database and repeated the same analysis. We found that none of the files carried genome build information in either their filename or as part of their header. Although these statistics (Table 2) largely stem from the fact that these file formats do not require genome build information as an obligatory field, this exemplifies the extent to which genome build information is detached from the genomic interval files after downloading them from public repositories. Once such genomic interval files are downloaded and exchanged between computers, one cannot totally exclude the possibility of failure to record the genome build information, thereby leading to additional time investment in

Table 2 Genome build information carried in filenames or header lines

\begin{tabular}{lllll}
\hline File & $\begin{array}{l}\text { Total } \\
\text { checked }\end{array}$ & $\begin{array}{l}\text { Only in } \\
\text { filename (\%) }\end{array}$ & $\begin{array}{l}\text { Only in the } \\
\text { file (\%) }\end{array}$ & $\begin{array}{l}\text { In both } \\
(\%)\end{array}$ \\
\hline GEO: BED & 967 & $149(15.4)$ & $213(22)$ & $84(8.7)$ \\
GEO: GFF & 2105 & 0 & 0 & $12(0.6)$ \\
ENCODE: BED & 26,503 & 0 & 0 & 0 \\
\hline
\end{tabular}

procuring the genome build information, or resulting in erroneous integrations.

\section{Examples of attaching genome build information to genomic track files}

The examined records that did store genome build information stored it in any of the following ways: (1) a header line (commented line) that specified genome build version (e.g. [12]); (2) as part of the 'track name', which is usually the header line that appears in the track files of genome browsers (e.g. [12]); (3) recorded the path of input files in the header, where the path contained genome build information (e.g. [13]); (4) made use of required fields, like 'source' or 'feature', to include genome build information (e.g. [14]); or (5) as part of the filenames [14]. Further, we found that customised file formats in some instances dedicated a column to store genome build version (e.g. [15]).

\section{Recommendation on ways to specify genome build}

To ensure that genome build information stays with the data, we recommend that the information is included as part of initial header/comment lines inside the file itself. For most file formats, a comment line starting with a hash (\#) would allow the information to be human readable, without disturbing parsers. This is explicitly supported, for example, in file formats like GFF [16] and also appears to be allowed de facto in formats like BED [17] (though not formally specified as part of the format [18]). For BED files, as an alternative, one could add 'genome $=\mathrm{xxx}^{\prime}$ ' as part of a 'track'-prefixed header that is allowed in the track files used by genome browsers [18]. In cases where adding information to the data contents of a file is not possible/practical, an alternative is to specify genome build as part of the file name. Although the file name may change as it moves between people and computers, it is often stable. Having the genome build only as part of the full path/URL of a file is risky, as it will be disrupted by almost any transfer. A last option is to simply specify the genome build for every data line along with the sequence (chromosome) identifier, although this is superfluous for datasets where all regions are from the same genome build.

\section{Tool to predict the genome build version}

Although not a substitute to explicitly supplementing the genome build information, we provide here a tool to predict the genome build version of orphan genomic track files. The tool is available at [19] on the Genomic HyperBrowser [20], an integrated open-source tool for statistical genome analysis. The web tool currently supports 19 species. In addition, we provide a commandline tool as an $\mathrm{R}$ package that supports human, mouse and Drosophila genome builds [21]. Both the web tool and the command-line tool accept any of the standard 
genomic file formats like BED, GFF, WIG, broadPeak and narrowPeak. As custom file formats do not formally define in which columns or fields the sequence names and coordinates are provided, standard file parsers cannot directly handle them. Therefore, other file formats need to be converted to any of the standard file formats prior to running the tools. When we tested the web tool on public data from ENCODE, the tool predicted the correct genome build for $98.2 \%$ of the broad peak files $(n=223)$ for the K562 cell line. We noticed that the tool failed when the genomic track files did not strictly adhere to the file format specifications (Additional file 1). Notably, any such tool cannot distinguish between genome builds if the genomic track files of interest do not contain sequence coordinates that are unique to a genome build version. In other words, the prediction is infeasible if all the input sequence coordinates are equally compatible with two or multiple genome builds.

\section{Conclusions}

This study demonstrates the detachment of genome build information from genomic track files in both public repositories and journal articles, which could lead to additional time investment in inferring the genome build or potential erroneous genome arithmetic operations. The findings also exemplify the extent of incompatibility between the sequence coordinates of two genome build versions that would result in erroneous integrations when performing genome arithmetic operations. We propose three ways to ensure that genome build information is always carried along with genomic track files, where the preferable solution is to record it as part of the header lines in genomic track files. To facilitate the adoption of orphan genomic track files, we provide a tool that predicts the genome build version.

\section{Additional file}

Additional file 1: Additional Tables S1 and S2. (PDF $79 \mathrm{~kb}$ )

\section{Abbreviations}

ENCODE: Encyclopedia of DNA Elements; GEO: Gene Expression Omnibus; UCSC: University of California Santa Cruz

\section{Acknowledgements}

We thank Sveinung Gundersen and Lex Nederbragt for helpful discussion.

\section{Funding}

Stiftelsen Kristian Gerhard Jebsen (K.G. Jebsen Coeliac Disease Research (entre).

\section{Availability of data and materials}

All the scripts used for web crawling, querying the databases, retrieving the files and all URLs of the files retrieved have been submitted to Zenodo database and can be accessed at [22]. The web tool to predict the genome build information is available at [19]. The R package is available at [21] and was also deposited to Zenodo database at [23]. All the software and scripts are available under open source licence Artistic 2.0.

\section{Authors' contributions}

CK and DD performed the database queries, web crawling, analyses and tool implementations. EH conceived part of the analyses and tool implementation. GKS conceived the idea and part of the analyses. CK and GKS drafted the manuscript. All authors read and approved the final manuscript.

\section{Competing interests}

The authors declare that they have no competing interests.

\section{Publisher's Note}

Springer Nature remains neutral with regard to jurisdictional claims in published maps and institutional affiliations.

\section{Author details}

${ }^{1}$ Department of Informatics, University of Oslo, 0316 Oslo, Norway. ${ }^{2}$ K.G. Jebsen Coeliac Disease Research Centre, University of Oslo, 0318 Oslo, Norway. ${ }^{3}$ Department of Tumor Biology, Institute for Cancer Research, Oslo University Hospital, 0424 Oslo, Norway. Institute for Cancer Genetics and Informatics, The Norwegian Radium Hospital, Oslo University Hospital, 0424 Oslo, Norway.

Received: 12 April 2017 Accepted: 29 August 2017

Published online: 14 September 2017

References

1. Eisenstein M. Big data: the power of petabytes. Nature. 2015;527:S2-4.

2. Barrett T, Wilhite SE, Ledoux P, Evangelista C, Kim IF, Tomashevsky M, et al. NCBI GEO: archive for functional genomics data sets - update. Nucleic Acids Res. 2013:41:D991-5.

3. Parkinson H, Sarkans U, Kolesnikov N, Abeygunawardena N, Burdett T, Dylag $M$, et al. ArrayExpress update-an archive of microarray and highthroughput sequencing-based functional genomics experiments. Nucleic Acids Res. 2011;39:D1002-4.

4. ENCODE Project Consortium. An integrated encyclopedia of DNA elements in the human genome. Nature. 2012;489:57-74.

5. Lek M, Karczewski KJ, Minikel EV, Samocha KE, Banks E, Fennell T, et al. Analysis of protein-coding genetic variation in 60,706 humans. Nature. 2016:536:285-91.

6. The FANTOM Consortium and the RIKEN PMI and CLST (DGT), Forrest AR, Kawaji H, Rehli M, Baillie JK, de Hoon MJ, et al. A promoter-level mammalian expression atlas. Nature. 2014;507:462-70.

7. Nellore A, Jaffe AE, Fortin J-P, Alquicira-Hernández J, Collado-Torres L, Wang $S$, et al. Human splicing diversity and the extent of unannotated splice junctions across human RNA-seq samples on the Sequence Read Archive. Genome Biol. 2016;17:266.

8. Walsh R, Thomson KL, Ware JS, Funke BH, Woodley J, McGuire KJ, et al. Reassessment of Mendelian gene pathogenicity using 7,855 cardiomyopathy cases and 60,706 reference samples. Genet Med. 2017;19: 192-203.

9. Brazma A, Hingamp P, Quackenbush J, Sherlock G, Spellman P, Stoeckert C, et al. Minimum information about a microarray experiment (MIAME)-toward standards for microarray data. Nat Genet. 2001;29:365-71.

10. FGED: MINSEQE. http://www.fged.org/projects/minsege/. Accessed 21 Aug 2017.

11. fetchChromSizes. http://hgdownload.cse.ucsc.edu/admin/exe/linux.x86_64/ fetchChromSizes. Accessed 21 Aug 2017.

12. Example of a bed file that contains genome build information. ftp://ftp.ncbi. nlm.nih.gov/geo/samples/GSM739nnn/GSM739245/suppl/GSM739245_CC_ G1_chipC_NCBlv36_signal_Hs_hg18.bed.gz.

13. Example of a bed file that stores genome build information as part of file path. ftp://ftp.ncbi.nlm.nih.gov/geo/samples/GSM1088nnn/GSM1088491/ suppl/GSM1088491_INA6_P_chipN_high.bed.gz.

14. Example of $\mathrm{gff}$ file that uses one of the required fields to store genome build information. ftp://ftp.ncbi.nlm.nih.gov/geo/samples/ GSM520nnn/GSM520528/suppl/GSM520528_HG18-1-179071_overlap_ 635_ratio_peaks.gff.gz.

15. Example of tsv file from ICGC portal that contains a dedicated field to specify genome build information. https://dcc.icgc.org/api/v1/ download?fn=/release_23/Projects/ALL-US/simple_somatic_mutation.open. ALL-US.tsv.gz. 
16. The Sequence-Ontology/Specifications. GFF3 file format. https://github. com/The-Sequence-Ontology/Specifications/blob/master/gff3.md Accessed 21 Aug 2017.

17. The Genome Browser Database. BED format treats \# character as comments. https://users.soe.ucsc.edu/ kent/gbd.html. Accessed 21 Aug 2017.

18. BED format. https://genome.ucsc.edu/FAQ/FAQformat\#format1.

19. Genomic HyperBrowser: Genome Build Predictor. https://hyperbrowser.uio. no/refgenome. Accessed 21 Aug 2017.

20. Sandve GK, Gundersen S, Rydbeck H, Glad I, Holden L, Holden M, et al. The Genomic HyperBrowser: inferential genomics at the sequence level. Genome Biol. 2010;11:R121.

21. GenomeBuildPredictor R package. https://github.com/KanduriC/ GenomeBuildPredictor. Accessed 21 Aug 2017.

22. Zenodo: Scripts and URLs used in database queries and web-crawling for article "Genome build information is an essential part of genomic track files". https://doi.org/10.5281/zenodo.438204. Accessed 21 Aug 2017.

23. Zenodo: GenomeBuildPredictor: R package that predicts the genome build version of genomic track files. https://doi.org/10.5281/zenodo.804687. Accessed 21 Aug 2017.

24. Example genomic track file from UCSC. http://hgdownload.cse.ucsc.edu/ goldenPath/hg19/encodeDCC/wgEncodeAwgDnaseMasterSites/. Accessed 21 Aug 2017.

25. Example genomic track file from ENCODE project database. https://www. encodeproject.org/files/ENCFF799KQV/@@download/ENCFF799KQV.bed.gz.

26. Example genomic track file of Roadmap Epigenomics. 1ftp://ftp.ncbi.nlm.nih. gov/geo/samples/GSM409nnn/GSM409307/suppl/GSM409307_UCSD.H1. H3K4me1.LL228.bed.gz.

27. Example genomic track file from GEO database. ftp://ftp.ncbi.n/m.nih.gov/ geo/samples/GSM2131nnn/GSM2131012/suppl/GSM2131012_AT3G45810 DTAF1_Dex_6h_narrowPeak_p16.bed.gz.

\section{Submit your next manuscript to BioMed Central} and we will help you at every step:

- We accept pre-submission inquiries

- Our selector tool helps you to find the most relevant journal

- We provide round the clock customer support

- Convenient online submission

- Thorough peer review

- Inclusion in PubMed and all major indexing services

- Maximum visibility for your research

Submit your manuscript at www.biomedcentral.com/submit 